\title{
Decoupled Nominal 2-D Direction-of -Arrival Estimation Algorithm for Coherently Distributed Source
}

\author{
Yinghua Han*, Jinkuan Wang and Qiang Zhao \\ College of Computer and Communication Engineering, Northeastern University at Qinhuangdao, Qinghuangdao 066004 P.R. China
}

Received: 19 Mar. 2013, Revised: 20 Jul. 2013, Accepted: 22 Jul. 2013

Published online: 1 Mar. 2014

\begin{abstract}
A computationally efficient method for nominal 2-D (azimuth and elevation) direction-of-arrival (DOA) estimation of coherently distributed source impinging on the far field is presented. Since the coherently distributed source is characterized by four parameters, the nominal azimuth DOA, angular spread of the nominal azimuth DOA, the nominal elevation DOA, and angular spread of the nominal elevation DOA, the computational complexity of the parameter estimation is normally high demanding. So a low complexity estimation algorithm is proposed in this paper, the key idea of which is to apply a subspace-based method without eigendecomposition in beamspace and a proposed second-order statistics for estimating the nominal elevation and azimuth DOAs. The proposed decoupled estimation algorithm does not involve any searching. It has a lower computational complexity particularly when the radio of array size to the number of source is large, at the expense of negligible performance loss. Simulation results are included to demonstrate the performance of the proposed technique.
\end{abstract}

Keywords: Array signal processing, coherently distributed source, 2-D DOA estimation, angular spread

\section{Introduction}

DOA estimation by array antenna is important in various applications including location information [1,2]. 2-D DOA also has played an important role in areas such as radar, sonar, radio astronomy, and mobile communication systems. So estimation of it has received a significant amount of attention over the last several decades $[3,4,5]$. Now the most popular techniques for 2-D DOA estimation have focused on sources that are modeled as points in space. However, in several applications, such a point source assumption can be irrelevant because signal scattering phenomena may result in angular spreading of source energy. In such cases, a distributed source model is more realistic than the point source one $[6,7,8]$.

In recent years, a number of investigators have proposed distributed source modeling, and parameter estimation techniques. For example, depending on the relationship between the channel coherently time and the observation period, the sources can be viewed either as coherently distributed or incoherently distributed $[9,10$, $11]$.

Some typical estimators have been proposed for azimuth-only estimation of the nominal DOA and angular spread of coherently or incoherently distributed source [9,
10,12,13,14]. All these methods are involved joint 2-D searching and computationally intensive. Some low-complexity estimators have also been given in $[15$, $16,17,18,19,20]$. In the low-complexity algorithms, some of them are sequential 1-D algorithms instead of joint 2-D searching $[16,17]$. Others are simpler but suboptimal solutions can be achieved by the subspace-based approach, which relies on signal subspace and noise subspace $[18,19,20]$.

However, for the problem of estimating the 2-D DOA, the distributed source is characterized by four parameters, the nominal azimuth DOA, angular spread of the nominal azimuth DOA, the nominal elevation DOA, and angular spread of the nominal elevation DOA, the computational complexity of parameter estimation is normally highly demanding. Simpler but suboptimal solutions can be achieved by SOS algorithm [16], which relies on eigendecomposition and 1-D searching for estimating the nominal azimuth DOA and elevation DOA. In this paper, we consider the coherently distributed source model and propose a low-complexity 2-D DOA estimation method using three uniform linear arrays. Based on the special array geometry and the relations between the signal subspaces, the nominal elevation DOA estimation can be

\footnotetext{
*Corresponding author e-mail: yhhan723@126.com
} 
obtained without eigenvalue decomposition. And then a second-order statistics is proposed for estimating the nominal azimuth DOA. In addition, we use beamspace transformation to reduce the computation, particularly in the situation where the radio of array size to the number of sources is large.

\section{Distributed Source Model}

Consider an array configuration which consists of three uniform linear subarrays with interspacing equal to a half wavelength of incident signals as Fig. 1. Let $X, Z$ and $W$ denotes the three subarrays and each linear array consists of $M$ elements.

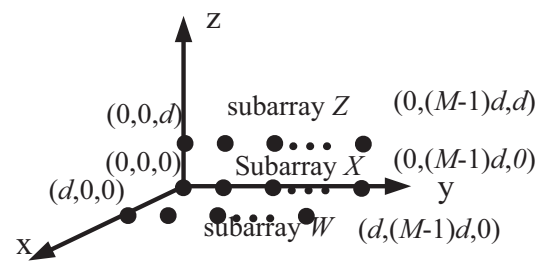

Fig. 1: The array configuration for 2-D DOA

Suppose that there are $q$ narrow-band sources impinging on the array. The received vector of subarray $X$ can be written as

$$
\boldsymbol{X}(t)=\sum_{i=1}^{q} \boldsymbol{S}_{i}(t)+\boldsymbol{n}_{X}(t)
$$

where $\boldsymbol{X}(t)$ is the array snapshot vector, $\boldsymbol{S}_{i}(t)$ is the vector that describes the contribution of the $i$ th signal source to the array output, and $\boldsymbol{n}_{X}(t)$ is additive zero-mean noise for subarray $X$ uncorrelated from the signals.

In point source modeling, the baseband signals of the $i$ th source is modeled as

$$
\boldsymbol{S}_{i}(t)=s_{i}(t) \boldsymbol{a}\left(\theta_{i}, \phi_{i}\right)
$$

where $s_{i}(t)$ is the complex envelope of the $i$ th source, and

$$
\begin{aligned}
& \boldsymbol{a}\left(\theta_{i}, \phi_{i}\right)=\left[1 \exp \left(-j 2 \pi(d / \lambda) \sin \theta_{1} \sin \phi_{1}\right) \cdots\right. \\
& \left.\exp \left(-j 2 \pi(M-1)(d / \lambda) \sin \theta_{i} \sin \phi_{i}\right)\right]^{\mathrm{T}}
\end{aligned}
$$

is the corresponding steering vector, $\theta_{i}$ and $\phi_{i}$ are the elevation DOA and azimuth DOA, respectively, $\lambda$ is the wavelength of the impinging signal.

In distributed source modeling, the source energy is considered to be spread over some angular volume. Hence, $\boldsymbol{S}_{i}(t)$ is written as

$$
S_{i}(t)=\iint \boldsymbol{a}(\vartheta, \varphi) \varsigma_{i}(\vartheta, \varphi, t) \mathrm{d} \vartheta \mathrm{d} \varphi
$$

where $\varsigma_{i}(\vartheta, \varphi, t)$ is the angular signal density of the $i$ th source and can be expressed as

$$
\varsigma_{i}(\vartheta, \varphi, t)=s_{i}(t) \ell_{i}\left(\vartheta, \varphi ; \boldsymbol{\mu}_{i}\right)
$$

under the coherently distributed source assumptions. In (4), $\ell(\vartheta, \varphi ; \boldsymbol{\mu})$ is a deterministic angular signal intensity function, and parametrized by the vector $\boldsymbol{\mu}=\left(\theta, \sigma_{\theta}, \phi, \sigma_{\phi}\right)$ denoting the nominal elevation DOA $\theta$, angular spread $\sigma_{\theta}$ of the nominal elevation DOA, the nominal azimuth DOA $\phi$, and angular spread $\sigma_{\phi}$.

The steering vector of subarray $X$ can be written as

$$
\boldsymbol{b}_{X}(\boldsymbol{\mu})=\iint \boldsymbol{a}(\vartheta, \varphi) \ell(\vartheta, \varphi ; \boldsymbol{\mu}) \mathrm{d} \vartheta \mathrm{d} \varphi
$$

As a common example of the coherently distributed source, assume that the deterministic angular signal intensity function $\ell(\vartheta, \varphi ; \boldsymbol{\mu})$ has the Gaussian shape as follows,

$$
\begin{aligned}
& \ell(\vartheta, \varphi ; \boldsymbol{\mu})=\left(1 /\left(2 \pi \sigma_{\theta} \sigma_{\phi}\right)\right) \times \\
& \quad \exp \left(-1 / 2\left((\vartheta-\theta)^{2} / \sigma_{\theta}^{2}+(\varphi-\phi)^{2} / \sigma_{\phi}^{2}\right)\right)
\end{aligned}
$$

The received signal vector in other subarrays $Z$ and $W$ can also be expressed as

$$
\begin{gathered}
\boldsymbol{Z}(t)=\sum_{i=1}^{q} \iint \boldsymbol{a}(\vartheta, \varphi) \ell(\vartheta, \varphi ; \boldsymbol{\mu}) \times \\
\exp (-j 2 \pi(d / \lambda) \cos \vartheta) s_{i}(t) \mathrm{d} \vartheta \mathrm{d} \varphi+\boldsymbol{n}_{Z}(t) \\
\boldsymbol{W}(t)=\sum_{i=1}^{q} \iint \boldsymbol{a}(\vartheta, \varphi) \ell(\vartheta, \varphi ; \boldsymbol{\mu}) \times \\
\quad \exp (-j 2 \pi(d / \lambda) \sin \vartheta \cos \varphi) s_{i}(t) \mathrm{d} \vartheta \mathrm{d} \varphi+\boldsymbol{n}_{W}(t)
\end{gathered}
$$

and the steering vectors are defined as $\boldsymbol{b}_{Z}(\boldsymbol{\mu})$ and $\boldsymbol{b}_{W}(\boldsymbol{\mu})$, respectively.

$$
\begin{gathered}
\boldsymbol{b}_{Z}(\boldsymbol{\mu})=\iint \boldsymbol{a}(\vartheta, \varphi) \times \\
\exp (-j 2 \pi(d / \lambda) \cos \vartheta) \ell(\vartheta, \varphi ; \boldsymbol{\mu}) \mathrm{d} \vartheta \mathrm{d} \varphi \\
\boldsymbol{b}_{W}(\boldsymbol{\mu})=\iint \boldsymbol{a}(\vartheta, \varphi) \times \\
\quad \exp (-j 2 \pi(d / \lambda) \sin \vartheta \cos \varphi) \ell(\vartheta, \varphi ; \boldsymbol{\mu}) \mathrm{d} \vartheta \mathrm{d} \varphi
\end{gathered}
$$

\section{Nominal 2-D DOA Estimation}

In general, an optimum estimation method for distributed sources can provide an excellent performance at the cost of intensive computation. Since the computational complexity increases dramatically with high dimensional parameters. It is noteworthy that a considerable simplification is possible by exploiting and utilizing the decoupling the nominal DOAs from that of angular spreads. 

as

$$
\begin{aligned}
& {\left[\boldsymbol{b}_{X}(\boldsymbol{\mu})\right]_{m}} \\
& =\iint \exp (-j 2 \pi(m-1)(d / \lambda) \sin \vartheta \sin \varphi) \times \\
& \left(1 /\left(2 \pi \sigma_{\theta} \sigma_{\phi}\right)\right) \times \\
& \exp \left(-1 / 2\left((\vartheta-\theta)^{2} / \sigma_{\theta}^{2}+(\varphi-\phi)^{2} / \sigma_{\phi}^{2}\right)\right) \mathrm{d} \vartheta \mathrm{d} \varphi
\end{aligned}
$$

where $[\cdot]_{m}$ indicates the $m$ th element of a vector. In distributed source, $\vartheta$ and $\varphi$ are all around $\theta$ and $\phi$. So with the change of variables $\vartheta-\theta=\tilde{\theta}$ and $\varphi-\phi=\tilde{\phi}, \tilde{\theta}$ and $\tilde{\phi}$ are small values. We can rewritten

$$
\begin{aligned}
& {\left[\boldsymbol{b}_{X}(\boldsymbol{\mu})\right]_{m}} \\
& \approx \iint \exp (-j 2 \pi(m-1)(d / \lambda)(\sin \theta+\tilde{\theta} \cos \theta) \times \\
& (\sin \phi+\tilde{\phi} \cos \phi)) \frac{1}{2 \pi \sigma_{\theta} \sigma_{\phi}} \\
& \exp \left(-1 / 2\left(\tilde{\theta}^{2} / \sigma_{\theta}^{2}+\tilde{\phi}^{2} / \sigma_{\phi}^{2}\right)\right) \mathrm{d} \tilde{\theta} \mathrm{d} \tilde{\phi} \\
& =\frac{1}{2 \pi \sigma_{\theta} \sigma_{\phi}} \exp (-j 2 \pi(m-1)(d / \lambda) \sin \theta \sin \phi) \times \\
& \int \exp (-j 2 \pi(m-1)(d / \lambda) \tilde{\phi} \sin \theta \cos \phi) \\
& \exp \left(-(1 / 2)\left(\tilde{\phi}^{2} / \sigma_{\phi}^{2}\right)\right) \mathrm{d} \tilde{\phi} \\
& \int \exp (-j 2 \pi(m-1)(d / \lambda) \tilde{\theta} \cos \theta \sin \phi) \\
& \exp \left(-(1 / 2)\left(\tilde{\theta}^{2} / \sigma_{\theta}^{2}\right)\right) \mathrm{d} \tilde{\theta}
\end{aligned}
$$

Let us consider the approximate closed form of $\boldsymbol{b}_{X}(\boldsymbol{\mu})$. Using the integral formula [21]

$$
\begin{aligned}
& \int_{-\infty}^{\infty} \exp \left(-f^{2} x^{2}\right) \exp [j p(x+\alpha)] \mathrm{d} x \\
& \quad=\sqrt{\pi} \exp \left(-p^{2} /\left(4 f^{2}\right)\right) \exp (j p \alpha) / f
\end{aligned}
$$

(12) can be expressed as

$$
\begin{aligned}
{\left[\boldsymbol{b}_{X}(\boldsymbol{\mu})\right]_{m} \approx } & \exp (-j 2 \pi(m-1)(d / \lambda) \sin \theta \sin \phi) \\
& \times\left[\boldsymbol{g}_{1}\right]_{m} \times\left[\boldsymbol{g}_{2}\right]_{m}
\end{aligned}
$$

where

$$
\begin{aligned}
& {\left[\boldsymbol{g}_{1}\right]_{m}=\exp \left(-2 \pi^{2}(m-1)^{2}(d / \lambda)^{2} \sin ^{2} \theta \cos ^{2} \phi \sigma_{\phi}^{2}\right),} \\
& {\left[\boldsymbol{g}_{2}\right]_{m}=\exp \left(-2 \pi^{2}(m-1)^{2}(d / \lambda)^{2} \cos ^{2} \theta \sin ^{2} \phi \sigma_{\theta}^{2}\right) .}
\end{aligned}
$$

In the matrix form it can be extended to

$$
\boldsymbol{b}_{X}(\boldsymbol{\mu})=\boldsymbol{a}(\boldsymbol{\theta}, \phi) \odot \boldsymbol{g}_{1} \odot \boldsymbol{g}_{2}
$$

where $\odot$ is the Schur-Hadamard or element product. $\boldsymbol{g}_{1}$ and $\boldsymbol{g}_{2}$ are real-valued because of the symmetry assumption on angular signal intensity.
For the steering vector $\boldsymbol{b}_{Z}(\boldsymbol{\mu})$, there is

$$
\begin{aligned}
& {\left[\boldsymbol{b}_{Z}(\boldsymbol{\mu})\right]_{m}} \\
& \approx \iint \exp (-j 2 \pi(m-1)(d / \lambda) \sin (\theta+\tilde{\theta}) \sin (\phi+\tilde{\phi})) \times \\
& \exp (-j 2 \pi(d / \lambda)(\cos \theta-\tilde{\theta} \sin \theta)) \times \\
& \frac{1}{2 \pi \sigma_{\theta} \sigma_{\phi}} \times \\
& \exp \left(-1 / 2\left((\vartheta-\theta)^{2} / \sigma_{\theta}^{2}+(\varphi-\phi)^{2} / \sigma_{\phi}^{2}\right)\right) \mathrm{d} \vartheta \mathrm{d} \varphi(16)
\end{aligned}
$$

Using $2 \pi(d / \lambda) \tilde{\theta} \approx 0, \boldsymbol{b}_{Z}(\boldsymbol{\mu})$ can be rewritten as

$$
\begin{aligned}
& {\left[\boldsymbol{b}_{Z}(\boldsymbol{\mu})\right]_{m}} \\
& \approx \iint \exp (-j 2 \pi(m-1)(d / \lambda) \sin (\theta+\tilde{\theta}) \sin (\phi+\tilde{\phi})) \times \\
& \exp (-j 2 \pi(d / \lambda) \cos \theta) \frac{1}{2 \pi \sigma_{\theta} \sigma_{\phi}} \times \\
& \exp \left(-1 / 2\left((\vartheta-\theta)^{2} / \sigma_{\theta}^{2}+(\varphi-\phi)^{2} / \sigma_{\phi}^{2}\right)\right) \mathrm{d} \vartheta \mathrm{d} \varphi \text { (17) }
\end{aligned}
$$

According to (14) and (17), we can write the following equations,

$$
\boldsymbol{b}_{Z}(\boldsymbol{\mu}) \approx \exp (-j 2 \pi(d / \lambda) \cos \theta) \boldsymbol{b}_{X}(\boldsymbol{\mu})
$$

For the steering vector $\boldsymbol{b}_{W}(\boldsymbol{\mu})$, we also have

$$
\boldsymbol{b}_{W}(\boldsymbol{\mu}) \approx \exp (-j 2 \pi(d / \lambda) \sin \theta \cos \phi) \boldsymbol{b}_{X}(\boldsymbol{\mu})
$$

From (18) and (19), the array response vector has the following expression,

$$
\left[\begin{array}{lll}
\boldsymbol{b}_{X} & \boldsymbol{b}_{Z} & \boldsymbol{b}_{W}
\end{array}\right]^{\mathrm{T}}=\left[\begin{array}{lll}
\boldsymbol{b}_{X} & \boldsymbol{b}_{X} \boldsymbol{\Phi}_{1} & \boldsymbol{b}_{X} \boldsymbol{\Phi}_{2}
\end{array}\right]^{\mathrm{T}}
$$

where

$$
\begin{aligned}
\boldsymbol{\Phi}_{1}= & \operatorname{diag}\left[\exp \left(-j 2 \pi(d / \lambda) \cos \theta_{1}\right)\right. \\
& \exp \left(-j 2 \pi(d / \lambda) \cos \theta_{2}\right) \cdots \\
& \left.\exp \left(-j 2 \pi(d / \lambda) \cos \theta_{q}\right)\right]
\end{aligned}
$$

and

$$
\begin{aligned}
\boldsymbol{\Phi}_{2}= & \operatorname{diag}\left[\exp \left(-j 2 \pi(d / \lambda) \sin \theta_{1} \cos \phi_{1}\right)\right. \\
& \exp \left(-j 2 \pi(d / \lambda) \sin \theta_{2} \cos \phi_{2}\right) \cdots \\
& \left.\exp \left(-j 2 \pi(d / \lambda) \sin \theta_{q} \cos \phi_{q}\right)\right]
\end{aligned}
$$

The array response vector can be partitioned into

$$
\begin{aligned}
& {\left[\begin{array}{lll}
\boldsymbol{b}_{X} & \boldsymbol{b}_{Z} \boldsymbol{b}_{W}
\end{array}\right]^{\mathrm{T}} } \\
= & {\left[\boldsymbol{b}_{X_{1}}^{\mathrm{T}} \boldsymbol{b}_{X_{2}}^{\mathrm{T}}\left(\boldsymbol{b}_{X_{1}} \boldsymbol{\Phi}_{1}\right)^{\mathrm{T}}\right.} \\
& \left.\left(\boldsymbol{b}_{X_{2}} \boldsymbol{\Phi}_{1}\right)^{\mathrm{T}}\left(\boldsymbol{b}_{X_{1}} \boldsymbol{\Phi}_{2}\right)^{\mathrm{T}}\left(\boldsymbol{b}_{X_{2}} \boldsymbol{\Phi}_{2}\right)^{\mathrm{T}}\right]^{\mathrm{T}}
\end{aligned}
$$

where $\boldsymbol{b}_{X_{1}}$ and $\boldsymbol{b}_{X_{2}}$ are sub-matices of dimension $q \times q$ and $(M-q) \times q$, respectively. 
Assume that we have a criterion function, which has the nominal azimuth and elevation DOAs as unknown parameters. When we estimate the two DOAs, we have to generally find low cost algorithms. Beamspace transformation is one way of reducing computation and sometimes improving the estimation accuracy. Here we use beamspace preprocessing, which offers reduction of computational cost, particularly when the ratio of array size to the number of sources is large.

Let $\boldsymbol{T}_{l}$ be a $M \times L$ matrix with orthogonal columns, i.e., $\boldsymbol{T}_{l}^{\mathrm{H}} \boldsymbol{T}_{l}=\boldsymbol{I}, L$ is the dimensional of beamspace data and $L<M$. Then, the beamspace transformation is defined by applying $\boldsymbol{T}_{l}$ to the snapshots $\boldsymbol{X}(t), \boldsymbol{W}(t)$ and $\boldsymbol{Z}(t)$. The corresponding transformed received data matrix will have the form,

$$
\begin{aligned}
& \boldsymbol{X}_{B}(t)=\boldsymbol{T}_{l}^{\mathrm{H}} \boldsymbol{X}(t) \\
& \boldsymbol{W}_{B}(t)=\boldsymbol{T}_{l}^{\mathrm{H}} \boldsymbol{W}(t) \\
& \boldsymbol{Z}_{B}(t)=\boldsymbol{T}_{l}^{\mathrm{H}} \boldsymbol{Z}(t)
\end{aligned}
$$

and

$$
\boldsymbol{T}_{l}=\left[\begin{array}{lll}
\boldsymbol{T}_{l}^{(l)}, & \cdots, & \boldsymbol{T}_{L}^{(l)}
\end{array}\right]
$$

where

$\boldsymbol{T}_{l}^{(l)}=\left[1, \exp \left(-j 2 \pi k_{l}^{(l)} / M\right), \cdots, \exp \left(-j 2 \pi k_{l}^{(l)}(M-1) / M\right)\right]^{\mathrm{T}}$ and $\left\{k_{m}^{(l)}\right\}_{m=1}^{L}$ are integers.

For coherently distributed source, the beamspace transformation of the steering vector is defined as follows,

$$
\begin{aligned}
\boldsymbol{b}_{B X} & =\boldsymbol{T}_{l}^{\mathrm{H}} \boldsymbol{b}_{X} \\
\boldsymbol{b}_{B Z} & =\boldsymbol{T}_{l}^{\mathrm{H}} \boldsymbol{b}_{Z} \\
\boldsymbol{b}_{B W} & =\boldsymbol{T}_{l}^{\mathrm{H}} \boldsymbol{b}_{W}
\end{aligned}
$$

According (20) and (24), we have

$$
\begin{aligned}
\boldsymbol{b}_{B Z} & =\boldsymbol{b}_{B X} \boldsymbol{\Phi}_{1} \\
\boldsymbol{b}_{B W} & =\boldsymbol{b}_{B X} \boldsymbol{\Phi}_{2}
\end{aligned}
$$

From (24) and (25), we can write

$$
\begin{aligned}
& {\left[\begin{array}{lll}
\boldsymbol{b}_{B X} & \boldsymbol{b}_{B Z} & \boldsymbol{b}_{B W}
\end{array}\right]^{\mathrm{T}}} \\
& =\left[\boldsymbol{b}_{B X_{1}}^{\mathrm{T}} \boldsymbol{b}_{B X_{2}}^{\mathrm{T}}\left(\boldsymbol{b}_{B X_{1}} \boldsymbol{\Phi}_{1}\right)^{\mathrm{T}}\right. \\
& \left.\left(\boldsymbol{b}_{B X_{2}} \boldsymbol{\Phi}_{1}\right)^{\mathrm{T}}\left(\boldsymbol{b}_{B X_{1}} \boldsymbol{\Phi}_{2}\right)^{\mathrm{T}}\left(\boldsymbol{b}_{B X_{2}} \boldsymbol{\Phi}_{2}\right)^{\mathrm{T}}\right]^{\mathrm{T}}
\end{aligned}
$$

where the rank of $\boldsymbol{b}_{B X_{1}}$ and $\boldsymbol{b}_{B X_{2}}$ are $q \times q$ and $(L-q) \times q$. we have

$$
\begin{aligned}
& \boldsymbol{P}^{\mathrm{H}} \boldsymbol{b}_{B X_{1}} \\
= & {\left[\boldsymbol{b}_{B X_{2}}^{\mathrm{T}}\left(\boldsymbol{b}_{B X_{1}} \boldsymbol{\Phi}_{1}\right)^{\mathrm{T}}\right.} \\
& \left.\left(\boldsymbol{b}_{B X_{2}} \boldsymbol{\Phi}_{1}\right)^{\mathrm{T}}\left(\boldsymbol{b}_{B X_{1}} \boldsymbol{\Phi}_{2}\right)^{\mathrm{T}}\left(\boldsymbol{b}_{B X_{2}} \boldsymbol{\Phi}_{2}\right)^{\mathrm{T}}\right]^{\mathrm{T}}
\end{aligned}
$$

where $\boldsymbol{P}$ denotes propagator.

We can partition $\boldsymbol{P}$ as follows

$$
\boldsymbol{P}^{\mathrm{H}}=\left[\begin{array}{lllll}
\boldsymbol{P}_{1}^{\mathrm{T}} & \boldsymbol{P}_{2}^{\mathrm{T}} & \boldsymbol{P}_{3}^{\mathrm{T}} & \boldsymbol{P}_{4}^{\mathrm{T}} \boldsymbol{P}_{5}^{\mathrm{T}}
\end{array}\right]^{\mathrm{T}}
$$

where the dimensions of $\boldsymbol{P}_{1}, \boldsymbol{P}_{2}, \boldsymbol{P}_{3}, \boldsymbol{P}_{4}$ and $\boldsymbol{P}_{5}$ are identical with the dimensions of $\boldsymbol{b}_{B X_{2}}, \boldsymbol{b}_{B X_{1}} \boldsymbol{\Phi}_{1}, \boldsymbol{b}_{B X_{2}} \boldsymbol{\Phi}_{1}$, $\boldsymbol{b}_{B X_{1}} \boldsymbol{\Phi}_{2}$ and $\boldsymbol{b}_{B X_{2}} \boldsymbol{\Phi}_{2}$.

From (27) and (28), we can write the following,

$$
\begin{aligned}
& \boldsymbol{P}_{1} \boldsymbol{b}_{B X_{1}}=\boldsymbol{b}_{B X_{2}} \\
& \boldsymbol{P}_{2} \boldsymbol{b}_{B X_{1}}=\boldsymbol{b}_{B X_{1}} \boldsymbol{\Phi}_{1} \\
& \boldsymbol{P}_{3} \boldsymbol{b}_{B X_{1}}=\boldsymbol{b}_{B X_{2}} \boldsymbol{\Phi}_{1} \\
& \boldsymbol{P}_{4} \boldsymbol{b}_{B X_{1}}=\boldsymbol{b}_{B X_{1}} \boldsymbol{\Phi}_{2} \\
& \boldsymbol{P}_{5} \boldsymbol{b}_{B X_{1}}=\boldsymbol{b}_{B X_{2}} \boldsymbol{\Phi}_{2}
\end{aligned}
$$

Using (29), we can write

$$
\begin{aligned}
& \boldsymbol{P}_{3} \boldsymbol{b}_{B X_{1}}=\boldsymbol{P}_{1} \boldsymbol{b}_{B X_{1}} \boldsymbol{\Phi}_{1} \\
& \boldsymbol{P}_{5} \boldsymbol{b}_{B X_{1}}=\boldsymbol{P}_{1} \boldsymbol{b}_{B X_{1}} \boldsymbol{\Phi}_{2}
\end{aligned}
$$

So we have

$$
\begin{aligned}
& \boldsymbol{P}_{1}^{\dagger} \boldsymbol{P}_{3} \boldsymbol{b}_{B X_{1}}=\boldsymbol{b}_{B X_{1}} \boldsymbol{\Phi}_{1} \\
& \boldsymbol{P}_{1}^{\dagger} \boldsymbol{P}_{5} \boldsymbol{b}_{B X_{1}}=\boldsymbol{b}_{B X_{1}} \boldsymbol{\Phi}_{2}
\end{aligned}
$$

where $\dagger$ denotes the pseudoinverse. This means that the estimation of the diagonal elements of matrices $\boldsymbol{\Phi}_{1}$ and $\boldsymbol{\Phi}_{2}$ can be obtained by finding the eigenvalues of $\boldsymbol{P}_{1}^{\dagger} \boldsymbol{P}_{3}$ and $\boldsymbol{P}_{1}^{\dagger} \boldsymbol{P}_{5}$.

Now, let $\boldsymbol{Y}_{B}(t)=\left[\begin{array}{lll}\boldsymbol{X}_{B}^{\mathrm{T}}(t) & \boldsymbol{W}_{B}^{\mathrm{T}}(t) & \boldsymbol{Z}_{B}^{\mathrm{T}}(t)\end{array}\right]^{\mathrm{T}}$, and $\hat{\boldsymbol{R}}_{Y Y}=E\left\{\boldsymbol{Y}_{B}(t) \boldsymbol{Y}_{B}^{\mathrm{H}}(t)\right\}$. An estimation of propagator $\boldsymbol{P}$ can be obtained by minimizing the cost functions,

$$
\hat{\boldsymbol{R}}_{Y Y}=\left[\begin{array}{ll}
\hat{\boldsymbol{G}} & \hat{\boldsymbol{H}}
\end{array}\right]
$$

where $\hat{\boldsymbol{G}}$ and $\hat{\boldsymbol{H}}$ are sub-matrices with dimension $3 L \times q$ and $3 L \times(3 L-q)$, respectively, and

$$
\begin{aligned}
& \hat{\boldsymbol{P}}=\|\boldsymbol{H}-\boldsymbol{G} \hat{\boldsymbol{P}}\|^{2} \\
& \hat{\boldsymbol{P}}=\left(\boldsymbol{G}^{\mathrm{H}} \boldsymbol{G}\right)^{-1} \boldsymbol{G}^{\mathrm{H}} \boldsymbol{H}
\end{aligned}
$$

Equation (20) shows that diagonal elements of $\boldsymbol{\Phi}_{1}$ and $\boldsymbol{\Phi}_{2}$ are related to the estimation of nominal elevation DOA and azimuth DOA as $\hat{\theta}$ and $\hat{\phi}$, where

$$
\begin{aligned}
& \hat{\boldsymbol{\theta}}=\arccos \left(\frac{\arg \left(\boldsymbol{\Phi}_{1}\right)}{-2 \pi(d / \lambda)}\right) \\
& \hat{\boldsymbol{\phi}}=\arccos \left(\frac{\arg \left(\boldsymbol{\Phi}_{2}\right)}{-2 \pi(d / \lambda) \sin \hat{\theta}}\right)
\end{aligned}
$$

The domain of arc-cosine function is $(-1,1)$. However, the absolute values of the above function arguments are often greater than 1 in mobile communication environments. Now assuming a single 
distributed source, we propose the following second-order statistics $r_{1}(k)$ to estimate the nominal azimuth DOA,

$$
\begin{aligned}
r_{1}(k)= & E\left(\boldsymbol{X}_{k+1}(t) \boldsymbol{X}_{k}^{*}\right) \\
= & E\left(s(t) s^{*}(t) \exp (-j 2 \pi(d / \lambda) \sin \theta \sin \boldsymbol{\phi})\right. \\
& \times\left[\boldsymbol{g}_{1}\right]_{k+1} \times\left[\boldsymbol{g}_{2}\right]_{k+1} \times\left[\boldsymbol{g}_{1}\right]_{k} \times\left[\boldsymbol{g}_{2}\right]_{k}
\end{aligned}
$$

where $k \in[1, M-1]$, and the estimation of the nominal elevation DOA and azimuth DOA can be obtained as

$$
\begin{aligned}
& \hat{\phi}=\arctan \left(\frac{\arg \left(r_{1}\right)}{\arg \left(\hat{\boldsymbol{\Phi}}_{2}\right)}\right) \\
& \hat{\theta}=\arctan \left(\frac{\left(\frac{\arg \left(\hat{\boldsymbol{\Phi}}_{2}\right)}{\arg \left(\hat{\boldsymbol{\Phi}}_{1}\right)}\right)}{\cos \hat{\boldsymbol{\phi}}}\right)
\end{aligned}
$$

Regarding the computational complexity, from (36), it is clear that the proposed method need not any peak-finding searching compared with SOS algorithm which has two successive 1-D searching. Furthermore, real-time implementation of signal subspace-based algorithms is impeded by the requirement for an $O\left(M^{3}\right)$ eigendecomposition. Recently, the beamspace approaches have decomposed the original data vector into several lower-dimensional beamspaces via a transformation, and then the parameter estimation is carried out based on the beamspace data. If the dimensional of beamspace data is $L<M$, the computational time involved by the estimation of the propagator from the data is in $O\left(q \times 3 L^{2}\right)$. So when the radio of array size to the number of sources is large, beamspace transformation can further reduce the computational effort.

\section{Simulation Results}

Computer simulations have been conducted to evaluate nominal 2-D nominal DOA estimation performance of the proposed method. The spacing between the two adjacent elements in any uniform linear array is set to a half wavelength of incoming signals.

In the first example, we compare the proposed algorithm with SOS method for a Gaussian-shaped distributed source at $\boldsymbol{\mu}=\left(70^{\circ}, 4^{\circ}, 30^{\circ}, 6^{\circ}\right)$ with three subarrays of 8 sensors. Further, 500 snapshots per trial and 500 independent trials in total are tested. We use root mean square error (RMSE), which is defined as $\sqrt{E\left\{(\widehat{\theta}-\theta)^{2}+(\widehat{\phi}-\phi)^{2}\right\}}$, as the performance measures. The RMSEs of the nominal elevation DOA and azimuth DOA estimated by the proposed method are illustrated at different SNR in Fig. 2. As it can be seen, the proposed algorithm has a substantially better estimation performance at low SNR. The explanation of this fact is that the second-order statistics has weaken the inflection of noise. Indeed, our technique gives biased estimates even at high values of SNR.

In the second example, we examine the estimation performance of the proposed algorithm with the influence of angular spread. Fig. 3 shows the RMSEs of the nominal $2-\mathrm{D}$ DOA when $\mathrm{SNR}=15 \mathrm{~dB}$, and angular spread varies. It is observed that the variation of the RMSEs in the proposed algorithm are rather small even when the angular spread increases. This is partly because of the proposed closed form solution of the nominal elevation and azimuth having little relationship with angular spread. The proposed method has better performance in the cases of small and large angular spreads.

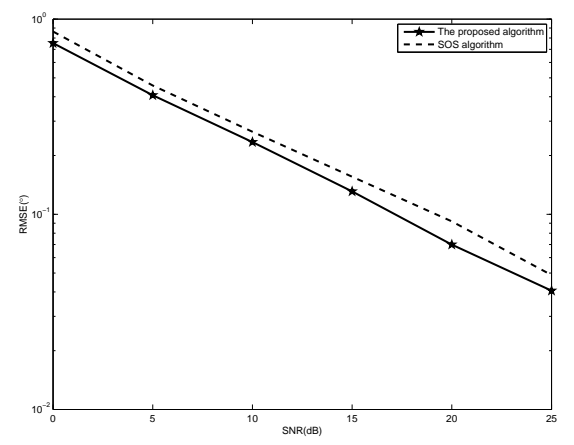

Fig. 2: RMSE for the nominal elevation DOA and azimuth DOA estimates versus SNR

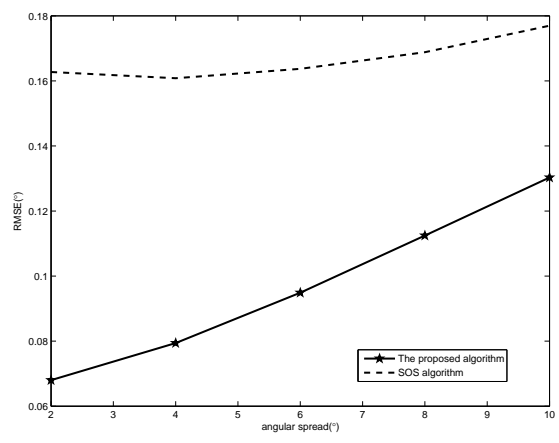

Fig. 3: RMSE for the nominal elevation DOA and azimuth DOA estimates versus angular spread

The influence of the number of snapshots is investigated in Fig. 4 for SNR $=15 \mathrm{~dB}$. It can be observed that the proposed algorithm presents effective performance even for a small number of snapshots.

\section{Conclusion}

In this paper, we have considered the modeling of coherently distributed source and the estimation of nominal 2-D DOA of distributed source. A 


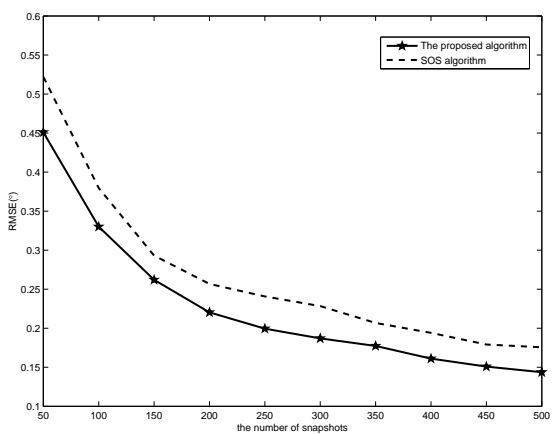

Fig. 4: RMSE for the nominal elevation DOA and azimuth DOA estimates versus the number of snapshots

low-complexity decoupled algorithm is proposed without any peak-finding searching. A subspace-based algorithm and a second-order statistics are proposed for the nominal elevation and azimuth DOAs. The proposed decoupled method has been shown to be useful in situations where the radio of array size to the number of source is large with a lower computational complexity.

\section{Acknowledgements}

This work is partially supported by the National Natural Science Foundation of China under Grant No.61104005, by Natural Science Foundation of Hebei Province under Grant No.F2011501052, by The central University Fundamental Research Foundation, under Grant. N110423004 and by Research Fund for the Doctoral Program of Higher Education of China under Grant No.20110042120015.

\section{References}

[1] A. Liu, G. Liao, C. Zeng, Z. Yang and Q. Xu, An eigenstructure method for estimating DOA and sensor gainphase errors, IEEE Trans. on Signal Processing, 59, 59445956 (2011)

[2] J. C. Hung and C. C. Llin, Hybridization of genetic algorithm and IBMUSIC applied to DOA estimation under coherent signals, Applied Mathematics \& Information Science, 6, 489S-496S (2012).

[3] Y. H. Ko, Y. J. Kim, H. I. Yoo, W. Y. Yang and Y. S. Cho, 2-D DOA estimation with cell searching for a mobile relay station with uniform circular array, IEEE Tran. on Communications, 58, 2805-2809 (2010).

[4] L. Gan, J. Gu and P. Wei, Estimation of 2-D DOA for noncircular sources using simultaneous SVD technique, IEEE Antennas and Wireless Propagation Letters, 7, 385388 ( 2008).
[5] B. Li, C. Peng and S. Biswas, Association of DOA estimation from two ULAs. IEEE Trans. Instrumentation and Measurement, 57, 1094-1101 (2008).

[6] Y. Xiong, G. Y. Zhang, B. Tang and H. Cheng, Blind identification and DOA estimation for array sources in presence of scattering, Journal of Systems Engineering and Electronics, 22, 393-397 (2011).

[7] C. T. Christou and G. M Jacyna, Simulation of the beam response of distributed dignals, IEEE Trans. Signal Processing, 53, 3023-3031 (2005).

[8] S. Shahbazpanahi and S. Valaee, A new approach to spatial power spectral density estimation for multiple incoherently distributed sources, Proc. 2007 ICASSP 1133-1136 (2007).

[9] A. Hassanien, S. Shahbazpanahi and A. B. Gershman, A generalized Capon estimator for localization of multiple spread sources, IEEE Trans Signal Processing, 52, 280-283 (2004).

[10] S. Valaee, B. Champagne and P. Kabal, Parameter localization of distributed sources, IEEE Trans. Signal Processing, 43, 2144-2153 (1995).

[11] R. Raich, J. Goldberg and H. Messor, Bearing estimation for a distributed source: modeling, inherent accuracy limitations and algorithm, IEEE Trans. Signal Processing, 48, 429-441 (2000).

[12] T. Trump and B. Ottersten, Estimation of nominal direction of arrival and angular spread using an array of sensors, IEEE Trans. Signal Processing, 45, 57-69 (1996).

[13] Y. Meng, P. Stoica and K. M. Wong, Estimation of the direction of arrival of spatially dispersed signals in array processing, IEE Proc.-F, 43, 1-9 (1996).

[14] J. Lee, J. Joung and J. D. Kim, A method for the direction-ofarrival estimation of incoherently distributed sources, IEEE Trans. on Vehicular Technology, 57, 2885-2893 (2008).

[15] S. Shahbazpanahi, S. Valaee and A. B. Gershman, A covariance fitting approach to parametric localization of multiple incoherently distributed sources, IEEE Trans. on Signal Processing, 52, 592-600 (2004).

[16] J. Lee, L. Song, H. Kwon and S. R. Lee, Low-complexity estimation of $2 D$ DOA for coherently distributed sources, Signal Processing, 1789-1802 (2003).

[17] M. Souden, S. Affes and J. Benesty, A two-stage approach to estimate the angles of arrival and the angular spreads of locally scatters sources, IEEE Trans. on Signal Processing, 56, 1968-1983 (2008).

[18] A. Zoubir, Y. Wang and P. Charge, spatially distributed sources localization with a subspace based estimator without eigendecomposition, Proc.2007 ICASSP, 10851088 (2007)

[19] S. Shahbazpanahi, S. Valaee and H. Bastani, Dsitributed source localization using ESPRIT algorithm, IEEE Trans. Signal Processing, 49, 2169-2178 (2001).

[20] A. Zoubir, Y. Wang and P. Charge, Efficient subspacebased estimator for localization of multiple incoherently distributed source, IEEE Trans. on Signal Processing, 56, 532-542 (2008).

[21] I. S. Gradshteyn and I. M. Ryzhik, Table of integrals, series, and products, Academic Press, Orlando, FL (1980). 


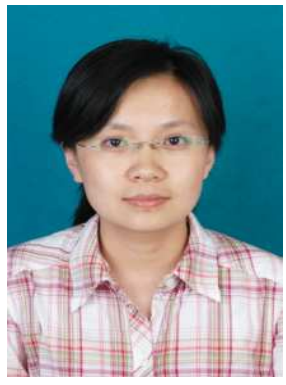

Yinghua Han received M.S. degree and Ph.D. degree in Navigation Guidance and Control in Northeastern University, China, in 2005 and 2008, respectively. She is currently a associate professor. Her research interests include array signal processing and mobile communication.

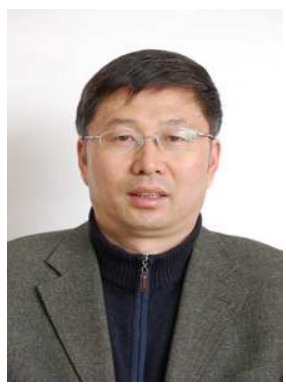

Jinkuan Wang was born on April 4, 1957 in Liaoning Province in China. He received the Ph.D. degree in Electronic Information and Communication Engineering in the University of Electro-Communications, Japan, in 1993. Since 1998, he has been with the college of information science and engineering, Northeastern University, China, where he is now a professor. His current research interests are in wireless communication, multiple antenna array communication systems and adaptive signal processing.

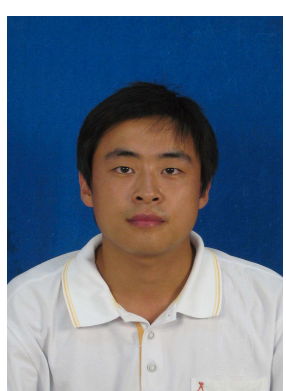

Qiang Zhao received M.S. degree in Control Theory and Control Engineering in Northeastern University, China, in 2010. He is currently a lecturer. His research interests include compressed sense and network communication. 